\title{
A debate model for cooperative agents to achieve common objectives
}

\author{
Abdelhak Imoussaten and Jacky Montmain \\ LGI2P, IMT Mines Ales, Univ Montpellier, Ales, France, firstname.name@mines-ales.fr
}

\begin{abstract}
We consider the situation where a group of agents is involved in the achievement of a common goal. Each agent owns a set of actions that are partial solutions for the problem to be solved. Different agents may have the same actions. In order to decide which action to select from the various agents, actions are assessed using a set of attributes. These attributes measure the extent to which the actions satisfy the common objective. The problem of action selection becomes more complex if we consider that agents do not necessarily know each others' actions, which complicates the coordination of joint actions. Interactions between agents may be affected by antagonistic personal interests. The more actions are selected from an agent, the greater his/her budget. Based on a Multiple Criteria Decision Analysis (MCDA) framework and a fuzzy model that links actions to the satisfaction of objectives, this paper examines the problem of collective selection of the necessary actions to achieve a goal. Only information necessary for the progress of the collective action is shared, and collective and personal goals coexist and are to be taken into account.
\end{abstract}

Keywords: Fuzzy sets, Multiple Criteria Decision Analysis, Debate modelling, Constraint programming, Collective choice.

\section{Introduction}

Several ways of working together can be used by agents, e.g., companies, algorithms, people, etc. to achieve a common goal. The interaction between the agents can take several forms. For instance, in a collaborative situation, the agents interact with each other on the basis of fully converging interests and goals. In a cooperative situation, agents do what they can to reach collective goals although they have also personal interests. The first type of interaction often occurs at inter-organizational level while the second often occurs at an intra-organizational level. In this paper we are interested in the cooperative situation. The advantages of this type of organisation are: the opportunity for an agent to achieve objectives that are unlikely with only his/her own resources, quicker market penetration and advanced technological innovations for companies, etc. But there are also disadvantages in the cooperative situation such as the lack of protection of the singularity of each company, the management of the distribution of control, risk equity, trust, etc. [3].

In this paper we will consider a simplified representation of cooperative agents. The main idea is to build joint actions from agents that achieve common objectives while limiting the disadvantages of a cooperative organization. More precisely, we try to limit the information shared by the agents whether concerning their weaknesses or their singularity. Indeed, only information necessary for the collective action is required. To further simplify, we consider that at interorganizational level, an agent has all the knowledge of his/her organisation and is able to make optimal propositions, i.e., a collaboration organisation is considered at the level of each agent.

Let us consider $m$ agents agent $_{1}, \ldots$, agent $m$ where each agent agent $_{l}, l \in \mathbb{L}=\{1, \ldots, m\}$ possesses a set of actions $\mathcal{A}_{l}$ and $\mathcal{A}=\bigcup_{l \in \mathbb{L}} \mathcal{A}_{l}$ the set of all actions of the agents. We consider that the actions are assessed within a Multiple Criteria Decision Analysis framework [9]. Several attributes are considered to determine the extent to which an objective is satisfied. Thus, we consider a set of $n$ attributes $\left\{X_{1}, \ldots, X_{n}\right\}$ where each attribute measures the extent to which the $i^{\text {th }}$ sub-objective $o_{i}$ is satisfied. The degree of satisfaction of the global objective is the aggregation of sub- 
objectives satisfaction degrees [5]. Furthermore a cost is associated to each action. Finally, the problem to be resolved is to select a subset of actions, also called an action plan, from the agents' actions under constraints such as respecting a predefined cost and a predefined degree of satisfaction. When the agents share all their knowledge before collective action, the problem can be stated as a multi-objective optimization problem [7]. When the number of actions is very large the resolution of this optimization problem clearly raises a combinatorial problem [10]. In the case of cooperative agents, where only partial knowledge is shared a debate model replaces the multi-objective optimization problem [8]. In this case agents share their partial knowledge at appropriate times.

This paper tries to establish a debate between the agents in order to build the action plan satisfying the common objectives. For this aim, we need: i) to choose a representation of the relationship between actions and the satisfaction of objectives; ii) to propose an organisation of the debate that corresponds to the cooperative hypothesis. In the proposed approach, for i) we consider that an agent has to provide only qualitative information in the form of ordinal values or linguistic terms. The information about the degree of satisfaction of an objective depends on the subjectivity of the agents so a qualitative setting is more appropriate. Furthermore, each action considered must allow the satisfaction of at least one objective, but it is not excluded that this action may have a negative impact on the other objectives. Thus, the scale used for the evaluation of each objective must be bivariate to enable positive and negative evaluation [6]. For ii), we consider a debate where the loss of earnings of the worst paid agent is minimized to avoid an unfair sharing of the allocated budget. Furthermore, we suppose that the agents do not necessarily know the capacities of the other agents, and that they have to learn from previous propositions in order to make appropriate subsequent propositions.

The paper is structured as follows. Section 2 gives the general principle for the debate structure regardless of the setting considered for the information concerning the action-objective relationship model. Sections 3 and 4 present the modelling of debate respectively in the ordinal and fuzzy sets settings. In the proposed debate model, only the learning step depends on the setting considered. In section 5 , we provide an illustration of our proposition.

\section{General principle for the debate structure}

As mentioned above, an operational cost is associated with each action plan $a p \subseteq \mathcal{A}$. We consider that the cost is a function $c: 2^{\mathcal{A}} \rightarrow \mathbb{R}^{+}$and we assume a linear cost model such as:

$$
c(a p)=\sum_{a \in a p} c(\{a\}), \forall a p \subseteq \mathcal{A}
$$

The solution ap should not exceed a predetermined budget denoted $\left.\left.b_{0} \in\right] 0, \infty\right]: c(a p) \leq b_{0}$. In addition, let $s_{N}(a p)$ denotes the degree of satisfaction of subobjectives in $N=\{1, \ldots, n\}$ by ap, a constraint on the degree of satisfaction of the objectives may be required: $s_{N}(a p) \geq \alpha_{0}$, where $\alpha_{0}$ is a predetermined threshold defined in the same scale as $s_{N}(a p)$. Nevertheless, agents may have preferences over cheaper solutions or solutions providing higher satisfaction. Let $\succ$ denote these preferences.

In collaborative approach where agents share all their knowledge of the system before collective action we obtain the following multi-objective optimization problem denoted $\operatorname{MOOP}\left(\mathcal{A}, N, \alpha_{0}, b_{0}\right)$.

$$
\begin{aligned}
& \max _{\succ}\left(s_{N}(a p), c(a p)\right) \quad \text { s.t: } \\
& \left\{\begin{array}{l}
a p \subseteq \mathcal{A}=\bigcup_{l \in \mathbb{L}} \mathcal{A}_{l} \\
s_{N}(a p) \geq \alpha_{0} \\
c(a p) \leq b_{0}
\end{array}\right.
\end{aligned}
$$

where $\max _{\succ}\left(s_{N}(a p), c(a p)\right)$ means the ap maximize the preference of the agent on the pair (satisfaction degree, cost). In the case of cooperative agents, the model is constructed gradually: each agent contributes when it is required, there is no a priori planning. The collective choice of action plan is thus modelled as a debate. The agents exchange knowledge and negotiate the way actions will be distributed. The more actions of an agent are carried out the greater his/her budget. Agents would propose their actions in order to increase their budget, then we need to manage the turn of propositions. This is done by selecting the agent having the best offer for the common objective at the current stage. When a proposal is made, the agent reveals the required information on the proposed actions. Each agent has to take advantage of this information to make subsequent successful propositions. This part is treated in Section 3 and 4 when the actions-objectives relationship model is presented.

Let us introduce the time variable $t$ explicitly in the notations. Let $a p_{t}$ be the common action plan build at time $t$ and $I_{t}$ the subset of satisfied objectives. The debate organization is broken down into the following steps: 
- At $t=0, a p_{0}=\emptyset$ and $I_{0}=\emptyset$.

- At $t \geq 1, a p_{t}$ denotes the current action plan and $I_{t}$ denotes the satisfied sub-objectives.

1. Each agent $l \in \mathbb{L}$ proposes a sub-action plan to complete $a p_{t-1}$. The new proposal should satisfy new sub-objectives in $N \backslash I_{t-1}: I_{t-1} \subset$ $I(t)$. The agent adopts a suitable strategy to make a proposal that maximizes his/her earnings (see subsection (2.1)).

2. A selection is made for the next action plan (see subsection 2.2);

3. Action plan $a p_{t}$ satisfying $I_{t}$ is built by adding new actions to $a p_{t}$;

4. Each agent updates his/her information about the proposed actions.

- When at $t \geq 1$ no proposal can be made by agents because of cost constraint, the debate starts from the beginning, i.e., the resulting common action plan is emptied, and agents update their information.

- The debate ends when $I_{t}=N$ and the cost constraint is respected.

Note that when a joint action plan satisfying all the objectives is found all other remaining actions are no longer receivable.

\subsection{Agent strategies}

At each step $t$, all the agents with available actions must adopt an appropriate strategy to propose the most relevant actions. The stake is twofold: i) the actions proposed by an agent must be chosen at time $t$ and, ii) the common action plan $a p_{t}$ must be successful.

The agent $l \in \mathbb{L}$ will use the optimisation problem (2) for $\operatorname{MOOP}\left(\mathcal{B}, I_{t}, \alpha_{0}, b_{0}\right)$, where $\mathcal{B}$ is constituted by his actions in $\mathcal{A}_{l}$ and the actions of other agents learned by agent $l$ within the debate. Let $A P_{t}^{l}$ be the Pareto front of solutions for the previous optimisation problem. Depending on the current stage, the following strategies may be applied by agent $l$ to select his proposition $s a p_{t}^{l}$ from the Pareto front $A P_{t}^{l}$ :

- $s a p_{t}^{l}$ may maximize the number of satisfied objectives;

- $s a p_{t}^{l}$ may maximize the satisfaction degree;

- $s a p_{t}^{l}$ may minimize the cost.

\subsection{Fair Sharing of Resources}

Using his/her strategy an agent $l \in \mathbb{L}$, with $A P_{t}^{l} \neq$ $\emptyset$, proposes a single sub-action plan $\operatorname{sap}_{t}^{l}$. Let $\mathbb{P}_{t}=$ $\left\{\operatorname{sap}_{t}^{l}, l \in \mathbb{L}: A P_{t}^{l} \neq \emptyset\right\}$ denote the set containing the subsequent propositions of the agents. This section proposes a fair resource sharing method to select the new proposition from $\mathbb{P}_{t}$.

Let $G_{l}\left(a p_{t-1}\right)=\sum_{a \in a p_{t-1} \cap \mathcal{A}_{l}} c(a)$ be the gain of $l$ from the common action plan $a p_{t-1}$. To quantify the loss of earnings of $l$ w.r.t $a p_{t-1}$ we can use the following formula:

$$
\rho\left(l, a p_{t-1}\right)=\left(G_{l}^{\max }-G_{l}\left(a p_{t-1}\right)\right) / G_{l}^{\max }
$$

where $G_{l}^{\text {max }}=\sum_{a \in \mathcal{A}_{l}} c(\{a\})$ is the maximal expected gain for $l$.

We consider in our approach that the group tries to avoid unfair sharing of the allocated budget by minimizing the loss of earnings of the worst-paid agent. The worst-paid agent w.r.t to $a p_{t-1}$ is defined as follows:

$$
l^{*}=\arg \max _{l}\left[\rho\left(l, a p_{t-1}\right)\right] .
$$

It follows that the new proposition $s a p_{t}^{l *} \in \mathbb{P}_{t}$ should verify:

$$
s a p_{t}^{l *}=\arg \min _{\operatorname{sap}_{t}^{l} \in \mathbb{P}_{t}} \max \left[\rho\left(l, s a p_{t}^{l} \cup a p_{t-1}\right)\right] .
$$

The new common action plan is $a p_{t}=s a p_{t}^{l *} \cup a p_{t-1}$.

\section{Ordinal setting}

\subsection{The action-objectives relationship}

The ordinal setting considered in this section is close to the one considered in [7] [8] [10]. Let us consider two ordinal scales $L_{s}=\left\{\alpha_{1}, \ldots, \alpha_{s-1}, \alpha_{s}\right\}$ where $0<\alpha_{1}<\ldots<\alpha_{s}$ and $L_{d}=\left\{\beta_{1}, \ldots, \beta_{d-1}, \beta_{d}\right\}$ where $0<\beta_{1}<\ldots<\beta_{d}$. To simplify, we consider the same bivariate scale for all objectives: $L_{s}$ contains the ordinal satisfaction degrees and $L_{d}$ contains the ordinal dissatisfaction, or negative impact, degrees on sub-objective $o_{i}, \forall i \in N=\{1, \ldots, n\}$.

The bivariate ordinal model describing the actionobjectives relationship can be represented by means of a digraph between the set of actions $\mathcal{A}$ and the set of sub-objectives $N$, such that (Fig. 1 shows an example): the arc between $a$ and $o_{i}$ is defined as:

$\operatorname{Arc}\left(a, o_{i}\right)=\left\{\begin{array}{l}+S_{i}(a) \text { in case of satisfaction } \\ -D_{i}(a) \text { in case of dissatisfaction } \\ \text { absence of arc } a \text { has no effect on } o_{i}\end{array}\right.$ 
where $S_{i}(a) \in L_{s}$ and $D_{i}(a) \in L_{d}$.

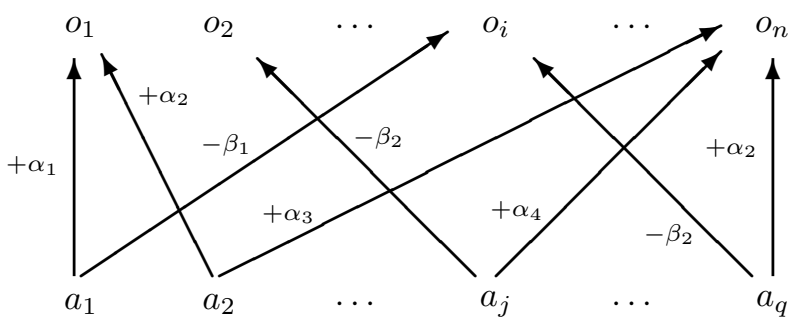

Figure 1: Digraph of actions-objectives relationships

Let us consider $a \in \mathcal{A}$ and $i \in N$. From formula 6 three situations are possible: 1) $a \in \mathcal{A}_{i}^{S}$ where $\mathcal{A}_{i}^{S} \subseteq \mathcal{A}$ is the subset of actions having a positive impact on $\left.o_{i} ; 2\right)$ $a \in \mathcal{A}_{i}^{D}$ where $\mathcal{A}_{i}^{D} \subseteq \mathcal{A}$ is the subset of actions having a negative impact on $\left.o_{i} ; 3\right) a$ has no effect on $o_{i}$.

To define the satisfaction degree and dissatisfaction degree of a subset of actions $a p \subseteq \mathcal{A}$ on objective $o_{i}$ from ordinal satisfaction degrees and dissatisfaction degrees of actions in ap only minimum, maximum operations and the combinations of these operations, e.g., median, are allowed.

The choice of aggregation functions for the satisfaction/dissatisfaction depends on the attitude of the agents regarding risk. We consider a pessimistic attitude defined as:

$$
S_{i}(a p)=\min _{a \in \mathcal{A}_{i}^{S} \cap a p} S_{i}(a)
$$

and

$$
D_{i}(a p)=\max _{a \in \mathcal{A}_{i}^{D} \cap a p} D_{i}(a)
$$

We consider that an action plan ap satisfies objective $o_{i}$ if there exists at least one action in $a p$ that improves $o_{i}$ and the degree of satisfaction is greater than the degree of dissatisfaction induced by $a p$. Let $s_{i}(a p)$ denote the degree of satisfaction of ap to objective $o_{i}$, we define $s_{i}(a p)$ as follows:

$s_{i}(a p)=\left\{\begin{array}{l}S_{i}(a p) \text { if } \mathcal{A}_{i}^{S} \cap a p \neq \emptyset \text { and } S_{i}(a p)>D_{i}(a p) \\ 0 \text { otherwise. }\end{array}\right.$

Finally, we define the satisfaction degree of ap for a subset of objectives $I \subseteq N$ as follows:

$$
s_{I}(a p)=\min _{i \in I} s_{i}(a p) .
$$

Note that more sophisticated aggregation operators such as Sugeno integral could be used in formula (10) but we would need additional information on agent preferences.

\subsection{The learning step in the debate model}

Agents then have to learn from the previous propositions in order to make appropriate subsequent propositions. At the beginning, the agents do not know the impacts induced by the actions of the other agents.

Let $l_{t}$ denote the agent selected at time $t$ to make proposal $\operatorname{sap}_{t}^{l} \subseteq \mathcal{A}_{l_{t}}$. Agents in $\mathbb{L} \backslash\left\{l_{t}\right\}$ do not know the satisfactions and dissatisfactions induced by actions in $s a p_{t}^{l}$. Agent $l_{t}$ must give the necessary information about his/her actions. The idea is as follows: If $a p_{t}=$ $s a p_{t}^{l} \cup a p_{t-1}$ is a new common action plan at time $t$ then any change in the calculus obtained by formulae (7) and (8) must be revealed to the other agents:

- case $S_{i}\left(\operatorname{sap}_{t}^{l}\right)<S_{i}\left(a p_{t-1}\right)$;

- case $D_{i}\left(s a p_{t}^{l}\right)>D_{i}\left(a p_{t-1}\right)$;

The satisfaction of $I_{t}$ by $a p_{t}$ and the construction of the satisfaction of $N \backslash I_{t}$ require the following:

- $\forall i \in I_{t}, S_{i}\left(a p_{t}\right)>D_{i}\left(a p_{t}\right)$ and $A_{i}^{S} \cap a p_{t} \neq \emptyset$ (reducing the satisfaction degree);

- $\forall i \in N \backslash I_{t}, A_{i}^{S} \cap a p_{t}=\emptyset$ or $\left[S_{i}\left(a p_{t}\right)<\alpha_{0}\right.$ and $\left.A_{i}^{D} \cap a p_{t}=\emptyset\right]$ (only the situation $\left[A_{i}^{S} \cap a p_{t} \neq \emptyset\right.$ and $A_{i}^{D} \cap a p_{t} \neq \emptyset$ and $\left.S_{i}\left(a p_{t}\right) \leq D_{i}\left(a p_{t}\right)\right]$ is not allowed over $N \backslash I_{t}$ ) (increasing the dissatisfaction degree).

Two cases are to be distinguished:

- first proposition: At $t=0$ the proposition of the agent $l_{0}$ is selected, he provides the impact of $s a p_{0}^{l}$ on each objective $o_{i}$ : For all $i \in N$,

- If $A_{i}^{S} \cap s a p_{0}^{l} \neq \emptyset, S_{i}\left(\operatorname{sap}_{0}^{l}\right)$ must be revealed,

- If $A_{i}^{D} \cap s a p_{0}^{l} \neq \emptyset, D_{i}\left(s a p_{0}^{l}\right)$ must be revealed,

- $c\left(s a p_{0}^{l}\right)$ must be revealed.

Each agent $l \in \mathbb{L} \backslash\left\{l_{0}\right\}$ learns the following information: For all $i \in N$,

- If $A_{i}^{S} \cap \operatorname{sap} p_{0}^{l} \neq \emptyset: \forall a \in \operatorname{sap}_{0}^{l}, S_{i}(a) \geq S_{i}\left(\operatorname{sap}_{0}^{l}\right)$

- If $A_{i}^{D} \cap \operatorname{sap}_{0}^{l} \neq \emptyset, \forall a \in \operatorname{sap}_{0}^{l}, D_{i}(a) \leq$ $D_{i}\left(\operatorname{sap}_{0}^{l}\right)$.

- $\forall i \in I_{0}, S_{i}\left(\operatorname{sap}_{0}^{l}\right)>D_{i}\left(\operatorname{sap}_{0}^{l}\right)$.

- The cost of $s a p_{0}^{l}$ is $c\left(s a p_{0}^{l}\right)$.

- At time $t \geq 1$, the common action plan is $a p_{t}=$ $s a p_{t}^{l} \cup a p_{t-1}$. For all $i \in N$ :

- If $A_{i}^{S} \cap a p_{t-1}=\emptyset$ and $A_{i}^{S} \cap s a p_{t}^{l} \neq \emptyset, S_{i}\left(\operatorname{sap}_{t}^{l}\right)$ must be revealed,

- If $S_{i}\left(s a p_{t}^{l}\right) \leq S_{i}\left(a p_{t-1}\right), S_{i}\left(s a p_{t}^{l}\right)$ must be revealed, 
- If $A_{i}^{D} \cap a p_{t-1}=\emptyset$ and $A_{i}^{D} \cap s a p_{t}^{l} \neq \emptyset, D_{i}\left(s a p_{t}^{l}\right)$ must be revealed,

- If $D_{i}\left(\operatorname{sap}_{t}^{l}\right) \geq D_{i}\left(a p_{t-1}\right), D_{i}\left(s a p_{t}^{l}\right)$ must be revealed,

$-c\left(\operatorname{sap}_{t}^{l}\right)$ must be revealed.

Each agent $l \in \mathbb{L} \backslash\left\{l_{t}\right\}$ learns the following information: For all $i \in N$,

- If $A_{i}^{S} \cap a p_{t-1}=\emptyset$ and $A_{i}^{S} \cap s a p_{t}^{l} \neq \emptyset$ : $\forall a \in \operatorname{sap}_{t}^{l}, S_{i}(a) \geq S_{i}\left(\operatorname{sap}_{t}^{l}\right)$.

- If $S_{i}\left(s a p_{t}^{l}\right) \leq S_{i}\left(a p_{t-1}\right)$ : $\forall a \in a p_{t-1}, S_{i}(a) \geq S_{i}\left(s a p_{t}^{l}\right)$.

- If $A_{i}^{D} \cap a p_{t-1}=\emptyset$ and $A_{i}^{D} \cap s a p_{t}^{l} \neq \emptyset$ : $\forall a \in \operatorname{sap}_{t}^{l}, D_{i}(a) \leq D_{i}\left(\operatorname{sap}_{t}^{l}\right)$,

- If $D_{i}\left(\operatorname{sap}_{t}^{l}\right) \geq D_{i}\left(a p_{t-1}\right)$ : $\forall a \in a p_{t-1}, D_{i}(a) \leq D_{i}\left(s a p_{t}^{l}\right)$.

- $\forall i \in I_{t}, S_{i}\left(a p_{t}\right)>D_{i}\left(a p_{t}\right)$.

- The cost of $s a p_{t}^{l}$ is $c\left(s a p_{t}^{l}\right)$.

\section{$4 \quad$ Fuzzy sets setting}

\subsection{The action-objectives relationship}

Let us consider two linguistic variables satisfaction and dissatisfaction to represent the impact of actions on objectives. We consider that the two variables take their values in $U=[0,1]$ in the continuous case and $U=\{0.1,0.2, \ldots, 1\}$ in the discrete. Furthermore, we consider the same linguistic terms for the two previous linguistic variables: low, medium, strong, extreme (see Figure 2).

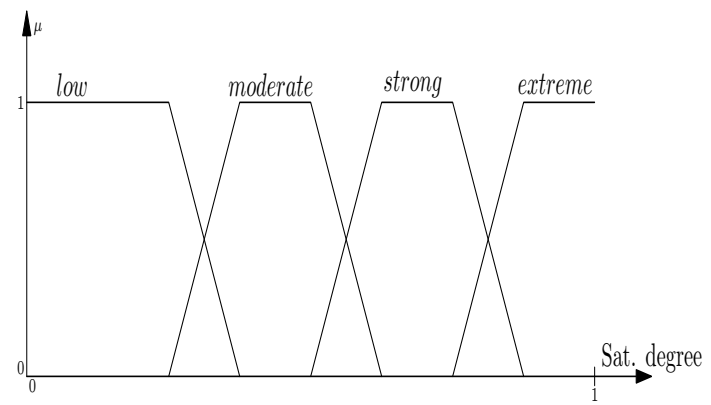

Figure 2: Linguistic variable associated to satisfaction degrees

In the case of a discrete universe of discourse, we consider the analogous terms to continuous ones:

Low $\equiv 1 / 0.1+1 / 0.2+0.9 / 0.3+0.6 / 0.4$.

Moderate $\equiv 0.1 / 0.2+0.2 / 0.3+0.8 / 0.4+1 / 0.5+$ $1 / 0.6+0.2 / 0.7$.

Strong $\equiv 0.1 / 0.5+0.5 / 0.6+1 / 0.7+0.9 / 0.8+0.1 / 0.9$. Extreme $\equiv 0.1 / 0.7+0.5 / 0.8+1 / 0.9+1 / 1$.
The bivariate fuzzy model can be represented through a digraph between $\mathcal{A}$ and $N$, such that (Fig. 3 shows an example): the arc between $a$ and $o_{i}$ is defined as follows:

$\operatorname{Arc}\left(a, o_{i}\right)=\left\{\begin{array}{l}+S_{i}^{a} \quad \text { in case of satisfaction } \\ -D_{i}^{a} \quad \text { in case of dissatisfaction } \\ \text { absence of arc } a \text { has no effect on } o_{i}\end{array}\right.$

Where $S_{i}^{a}$ and $D_{i}^{a}$ are linguistic terms with membership functions $\mu_{S_{i}^{a}}$ and $\mu_{D_{i}^{a}}$ respectively.

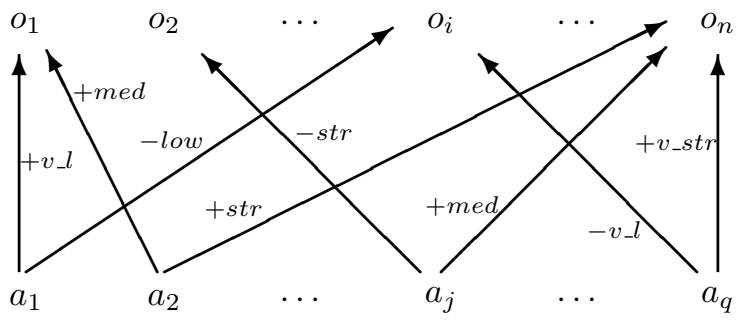

Figure 3: Digraph of actions-objectives relationships: $v_{-} l:=$ very low; $v_{s} s t r:=$ very strong; med:=medium; str: $=$ strong.

The quantities defined in the formulae 7 and 8 can be adapted for the fuzzy sets setting as follows:

$$
\begin{aligned}
& \mu_{S_{i}^{a p}}=\bigwedge_{a \in A_{i}^{S}(a p)} \mu_{S_{i}^{a}} ; \\
& \mu_{D_{i}^{a p}}=\bigvee_{a \in A_{i}^{D}(a p)} \mu_{D_{i}^{a}} .
\end{aligned}
$$

Let us consider an action plan $a p$ and an objective $o_{i}$. We consider the center of the defuzzification area to determine the satisfaction and dissatisfaction degree of $o_{i}$ in the continuous case:

1. $S_{i}(a p)=\frac{\int y \times \mu_{S_{i}^{a p}}(y) d y}{\int \mu_{S_{i}^{a p}}(y) d y}$ and

2. $D_{i}(a p)=\frac{\int y \times \mu_{D_{i}^{a p}}(y) d y}{\int \mu_{D_{i}^{a p}}(y) d y}$.

In the case of discrete universe of discourse, we consider the following defuzzification:

1. $S_{i}(a p)=\max _{y \in U} \min \left(y, \mu_{S_{i}^{a p}}(y)\right)$ and

2. $D_{i}(a p)=\max _{y \in U} \min \left(y, \mu_{D_{i}^{a p}}(y)\right)$.

We define the satisfaction degree of $o_{i}$ by $a p$ as in formula 9: 


$$
s_{i}(a p)=\left\{\begin{array}{l}
S_{i}(a p) \text { if } S_{i}(a p)>D_{i}(a p) \\
0 \text { otherwise }
\end{array}\right.
$$

We can also define the satisfaction and dissatisfactions of $a p$ on a subset of objectives $I \subseteq N$ by fixing the results of ap on the remaining objectives $N \backslash I$, at a given values. This is done by using the principle extension of Zadeh. $\forall I \subseteq N, \forall y \in[0,1]$ :

$$
\begin{gathered}
\mu_{S_{I}^{a p}}(y)=\bigvee_{\substack{y_{I} \in[0,1]^{|I|} \\
\phi_{S}\left(y_{I}, y_{N \backslash I}^{0}\right)=y}} \bigwedge_{i \in I} \mu_{S_{i}^{a p}}\left(y_{i}\right) . \\
\mu_{D_{I}^{a p}}(y)=\bigvee_{\substack{y_{I} \in[0,1]^{|I|} \\
\phi_{D}\left(y_{I}, z_{N \backslash I}^{0}\right)=y}} \bigwedge_{i \in I} \mu_{D_{i}^{a p}}\left(y_{i}\right) .
\end{gathered}
$$

where the notation $y_{I}$ represents the components of the vector $\left(y_{1}, \ldots, y_{n}\right)$ for the indices in $I$ and the notation $\left(y_{I}, y_{N \backslash I}^{0}\right)$ represents the vector $z$ where $z_{i}=y_{i}$ if $i \in I$ and $z_{i}=y_{i}^{0}$ if $i \in N \backslash I$. Operators $\phi_{S}$ and $\phi_{D}$ are two aggregation operators. For example, we can consider the weighted average operator for both operators or more sophisticated operators as fuzzy integrals.

Let us consider an action plan $a p$, a subset of objectives $I \subseteq N$ and two thresholds $\left.\left.\alpha_{I}, \beta_{I} \in\right] 0,1\right]$. We consider that ap satisfies objectives in $I$ if:

1. $s_{I}(a p)=\frac{\int y \times \mu_{S_{I}^{a p}}(y) d y}{\int \mu_{S_{I}^{a p}}(y) d y} \geq \alpha_{I}$ and

2. $d_{I}(a p)=\frac{\int y \times \mu_{D_{I}^{a p}}(y) d y}{\int \mu_{D_{I}^{a p}}(y) d y} \leq \beta_{I}$.

If the two previous conditions are satisfied, $s_{I}(a p)$ is considered as the degree of satisfaction of objectives on $I$ by $a p$, otherwise $s_{I}(a p)=0$.

\subsection{The learning step in the debate model}

For simplification purpose we consider the case of discrete linguistic variables.

In an analogue way as in subsection 3.2 , the construction of the common action plan requires that the agents propositions have to met the condition of formula 14 for all satisfied sub-objectives.

We recall the notation used in subsection 3.2. Let $l_{t}$ denotes the agent selected at time $t$ to make the proposal $\operatorname{sap}_{t}^{l} \subseteq \mathcal{A}_{l_{t}}$. Agents in $\mathbb{L} \backslash\left\{l_{t}\right\}$ don't know the satisfactions and dissatisfactions induced by actions in $s a p_{t}^{l}$. Any change induced by $s a p_{t}^{l}$ in the calculus obtained by formulae (12) and (13) must be revealed to the other agents:
- case $S_{i}\left(s a p_{t}^{l}\right)<S_{i}\left(a p_{t-1}\right)$;

- case $D_{i}\left(s a p_{t}^{l}\right)>D_{i}\left(a p_{t-1}\right)$;

The satisfaction of $I_{t}$ by $a p_{t}$ and the construction of the satisfaction of $N \backslash I_{t}$ require the following conditions as in subsection 3.2:

- $\forall i \in I_{t}, S_{i}\left(a p_{t}\right)>D_{i}\left(a p_{t}\right)$ and $A_{i}^{S} \cap a p_{t} \neq \emptyset$;

- $\forall i \in N \backslash I_{t}, A_{i}^{S} \cap a p_{t}=\emptyset$ or $\left[S_{i}\left(a p_{t}\right)<\alpha_{0}\right.$ and $\left.A_{i}^{D} \cap a p_{t}=\emptyset\right]$ (only the situation $\left[A_{i}^{S} \cap a p_{t} \neq \emptyset\right.$ and $A_{i}^{D} \cap a p_{t} \neq \emptyset$ and $\left.S_{i}\left(a p_{t}\right) \leq D_{i}\left(a p_{t}\right)\right]$ is not allowed over $N \backslash I_{t}$ );

Two cases are to be distinguished:

- first proposition: At $t=0$ the proposition of the agent $l_{0}$ is selected, he provides the impact of $\operatorname{sap}_{0}^{l}$ on each objective $o_{i}$ : For all $i \in N$,

- If $A_{i}^{S} \cap s a p_{0}^{l} \neq \emptyset, S_{i}\left(\operatorname{sap}_{0}^{l}\right)$ must be revealed,

- If $A_{i}^{D} \cap s a p_{0}^{l} \neq \emptyset, D_{i}\left(\operatorname{sap}_{0}^{l}\right)$ must be revealed,

- $c\left(s a p_{0}^{l}\right)$ must be revealed.

Each agent $l \in \mathbb{L} \backslash\left\{l_{0}\right\}$ learns the following information about $\mu_{S_{i}^{a p_{t}}}$ and $\mu_{D_{i}^{a p_{t}}}$ : For all $i \in N$,

- If $A_{i}^{S} \cap \operatorname{sap}_{0}^{l} \neq \emptyset$ then $\forall y \in U$

* if $y \geq S_{i}\left(\operatorname{sap}_{0}^{l}\right), \mu_{S_{i}^{s a p_{0}^{l}}}(y) \leq S_{i}\left(\operatorname{sap}_{0}^{l}\right)$;

* if $y<S_{i}\left(\operatorname{sap}_{0}^{l}\right), \mu_{S_{i}^{s_{0} p_{0}^{l}}}(y) \in U$ (no additional information can be obtained).

- If $A_{i}^{D} \cap \operatorname{sap}_{0}^{l} \neq \emptyset$ then $\forall y \in U$

* if $y \geq D_{i}\left(\operatorname{sap}_{0}^{l}\right), \mu_{D_{i}^{s a p_{0}^{l}}}(y) \leq D_{i}\left(\operatorname{sap}_{0}^{l}\right)$;

$*$ if $y<D_{i}\left(\operatorname{sap}_{0}^{l}\right), \mu_{D_{i}^{s a p_{0}}}(y) \in U$.

- $\forall i \in I_{0}, S_{i}\left(\operatorname{sap}_{0}^{l}\right)>D_{i}\left(\operatorname{sap}_{0}^{l}\right)$.

- The cost of $s a p_{0}^{l}$ is $c\left(s a p_{0}^{l}\right)$.

- At time $t \geq 1$, the common action plan is $a p_{t}=$ $\operatorname{sap}_{t}^{l} \cup a p_{t-1}$. For all $i \in N$ :

1. If $A_{i}^{S} \cap a p_{t-1}=\emptyset$ and $A_{i}^{S} \cap s a p_{t}^{l} \neq \emptyset, S_{i}\left(s a p_{t}^{l}\right)$ must be revealed,

2. If $S_{i}\left(s a p_{t}^{l}\right) \leq S_{i}\left(a p_{t-1}\right), S_{i}\left(s a p_{t}^{l}\right)$ must be revealed,

3. If $A_{i}^{D} \cap a p_{t-1}=\emptyset$ and $A_{i}^{D} \cap s a p_{t}^{l} \neq \emptyset, D_{i}\left(\operatorname{sap}_{t}^{l}\right)$ must be revealed,

4. If $D_{i}\left(s a p_{t}^{l}\right) \geq D_{i}\left(a p_{t-1}\right), D_{i}\left(s a p_{t}^{l}\right)$ must be revealed,

5. $c\left(s a p_{t}^{l}\right)$ must be revealed.

Each agent $l \in \mathbb{L} \backslash\left\{l_{t}\right\}$ learns the following information about $\mu_{S_{i}^{a p_{t}}}$ and $\mu_{D_{i}^{a p_{t}}}$ : For all $i \in N$,

- From 1: $\forall y \in U: y \geq S_{i}\left(\operatorname{sap}_{t}^{l}\right)$, $\mu_{S_{i}^{s a p_{t}^{l}}}(y) \leq S_{i}\left(\operatorname{sap}_{t}^{l}\right)$. 
- From 2: $\forall a \in \operatorname{sap}_{t}^{l}, S_{i}(a) \geq S_{i}\left(\operatorname{sap}_{t}^{l}\right)$.

- From 3: $\forall y \in U: y \geq D_{i}\left(\operatorname{sap}_{t}^{l}\right)$, $\mu_{D_{i}^{s a p_{t}^{l}}}(y) \leq D_{i}\left(\operatorname{sap}_{0}^{l}\right)$.

- From 4: $\forall a \in \operatorname{sap}_{t}^{l}, D_{i}(a) \leq D_{i}\left(\operatorname{sap}_{t}^{l}\right)$.

- From 5: The cost of $\operatorname{sap}_{t}^{l}$ is $c\left(\operatorname{sap}_{t}^{l}\right)$.

- $\forall i \in I_{t}, S_{i}\left(a p_{t}\right)>D_{i}\left(a p_{t}\right)$.

\section{Illustrations}

The case study concerns a simple manufacturing facility. The overall objective of the company is to increase its customer satisfaction. Four criteria are identified by the company to assess this overall objective w.r.t customer's satisfaction: Range of Products $\left(o_{1}\right)$, Product Pricing $\left(o_{2}\right)$, Product Quality $\left(o_{3}\right)$ and Delivery Time $\left(o_{4}\right)$. They are completed by an internal criterion: Social Climate $\left(o_{5}\right)$. In addition, actions are defined corresponding to the setting up of industrial performance improvement methods (the detail of these actions cannot be developed here for obvious reasons with regard to the length of the paper). We consider that actions have the same cost: $c(a)=1, \forall a \in \mathcal{A}$. We set $\alpha_{0}=0.2$ and the allocated budget at $b_{0}=4$. We consider in our simulation an ordinal setting to illustrate the updating step of the debate.

Tables 1, 2 and 3 give details about actions and objectives relationships. We consider $L_{S}=L_{D}=$ $\{0.1,0.2, \ldots, 1\}$.

\begin{tabular}{|c|c|c|c|c|}
\hline & \multicolumn{4}{|c|}{ agent 1} \\
\hline$o_{i}$ & $a_{1}$ & $a_{2}$ & $a_{3}$ & $a_{4}$ \\
\hline$o_{1}$ & -.2 & .9 & & -.3 \\
\hline$o_{2}$ & .8 & .2 & .3 & .6 \\
\hline$o_{3}$ & .5 & .5 & .8 & .2 \\
\hline$o_{4}$ & -.2 & -.5 & -.5 & .5 \\
\hline$o_{5}$ & & .6 & .7 & -.4 \\
\hline
\end{tabular}

Table 1: The impacts of agent 1 actions on subobjectives

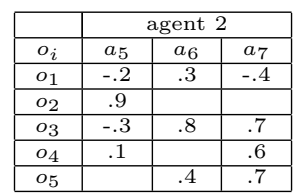

Table 2: The impacts of agent 2 actions on subobjectives

At the beginning, $t=0$, the three agents are the worst paid agents: from formula $(3) \rho(l, \emptyset)=1)$ for $l=1,2,3$. The three agent are asked to make a proposition. The resolution of the optimisation problem of formula 2 applied to the actions of each agents, we get three propositions composed by two actions. But agent 3 improves the maximum number of subobjectives with his actions $\left\{a_{9}, a_{10}\right\}$. Thus he proposes

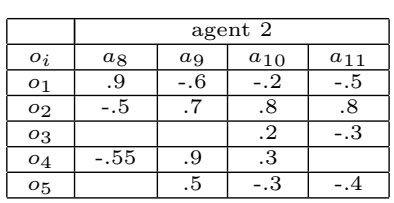

Table 3: The impacts of agent 3 actions on subobjectives

the first sub-action plan $s a p_{0}^{3}=\left\{a_{9}, a_{10}\right\}$. The proposition satisfy the sub-objectives $\left\{o_{2}, o_{3}, o_{4}, o_{5}\right\}$. Only the single sub-objective $o_{1}$ is still not yet satisfied.

Using the updating step of the subsection 3.2, Agent 1 , for example, has the following information (Table 4) (" $D$ none" means that the subaction plan has no negative impact and "S none" means means that the subaction plan has no positive impact).

\begin{tabular}{|c|c|c|c|c|c|c|}
\hline & \multicolumn{3}{|c|}{ agent 1} & \multicolumn{2}{|c|}{ agent 3} \\
\hline$o_{i}$ & $a_{1}$ & $a_{2}$ & $a_{3}$ & $a_{4}$ & $a_{9}$ & $a_{10}$ \\
\hline$o_{1}$ & -.2 & .9 & & -.3 & $\begin{array}{c}D \leq .6 \text { or } D \text { none } \\
S \text { none }\end{array}$ & $\begin{array}{c}D \leq .6 \text { or } D \text { none } \\
S \text { none }\end{array}$ \\
\hline$o_{2}$ & .8 & .2 & .3 & .6 & $\begin{array}{c}D \text { none } \\
S \geq .7 \text { or } S \text { none }\end{array}$ & $\begin{array}{c}D \text { none } \\
S \geq .7 \text { or } S \text { none }\end{array}$ \\
\hline$o_{3}$ & .5 & .5 & .8 & .2 & $\begin{array}{c}D \text { none } \\
S \geq .2 \text { or } S \text { none }\end{array}$ & $\begin{array}{c}D \text { none } \\
S \geq .2 \text { or } S \text { none }\end{array}$ \\
\hline$o_{4}$ & -.2 & -.5 & -.5 & .5 & $\begin{array}{c}D \text { none } \\
S \geq .3 \text { or } S \text { none }\end{array}$ & $\begin{array}{c}D \text { none } \\
S \geq .3 \text { or } S \text { none }\end{array}$ \\
\hline$o_{5}$ & & .6 & .7 & -.4 & $\begin{array}{c}D \leq .3 \text { or } D \text { none } \\
S \geq .5 \text { or } S \text { none }\end{array}$ & $\begin{array}{c}D \leq .3 \text { or } D \text { none } \\
S \geq .5 \text { or } S \text { none }\end{array}$ \\
\hline
\end{tabular}

Table 4: Information updating by agent 1

Only three actions have positive impacts on $o_{1}: a_{2}$ from Agent 1, $a_{6}$ from Agent 2 and $a_{8}$ from Agent 3. The worst-paid agents are Agents 1 and 2 but they have both the same loss and one action to add (see formula 5). However, if actions $\left\{a_{9}, a_{10}\right\}$ are in the common action plan, $a_{2}$ and $a_{6}$ can not be added: $\mathbb{P}_{1}=$ $\left\{\operatorname{sap}_{1}^{l}, l \in \mathbb{L}: A P_{1}^{l} \neq \emptyset\right\}=\emptyset$. It can be seen int Table 4 for agent 1 , no solution can be found in the front of Pareto. Thus agent 3 has to remove one of the two actions of the common action plan. If $a_{10}$ is removed then agent 1 makes the second proposition and $\left\{a_{2}, a_{9}\right\}$ improve all the sub-objectives and the debate ends. Otherwise, if $a_{9}$ is removed then agent 2 makes the second proposition and $\left\{a_{6}, a_{10}\right\}$ improve all the sub-objectives and the debate ends. Table 5 gives the steps of the debate when Agent 1 makes the second proposition.

\begin{tabular}{|c|c|c|c|c|c|c|c|c|c|c|}
\hline & \multicolumn{2}{|c|}{$o_{1}$} & \multicolumn{2}{c|}{$o_{2}$} & \multicolumn{2}{c|}{$o_{3}$} & \multicolumn{2}{c|}{$o_{4}$} & \multicolumn{2}{|c|}{$o_{5}$} \\
\hline & $S$ & $D$ & $S$ & $D$ & $S$ & $D$ & $S$ & $D$ & $S$ & $D$ \\
\hline$t=0$ & & .6 & .7 & & .2 & & .3 & & 0.5 & 0.3 \\
$\left\{a_{9}, a_{10}\right\}$ & & & & & & & & & & \\
\hline$t=1$ & & .6 & .7 & & & & .9 & & 0.5 & \\
$\left\{a_{9}\right\}$ & & & & & & & & & & \\
\hline$\{=2$ \\
$\left\{a_{2}, a_{9}\right\}$ & .9 & .6 & .7 & & .5 & & .9 & -.5 & 0.5 & \\
\hline
\end{tabular}

Table 5: Knowledge updating during the debate 


\section{Conclusion}

This paper has proposed a decision support system to help a group of cooperative agents to collectively design an action plan to satisfy a global common objective. The debate simulation is of interest for various reasons, it can be considered as an alternative to global optimization problem and it may also be envisaged as a decision-support system by a particular agent.

Various steps are introduced in the debate principles, minimizing the loss of earnings, group strategies and individual strategy, etc. They provide a globally rational model that enables a broad class of problems to be solved. This class of problem may be enlarged by providing other criteria with new semantics. May be it will be interesting to make connection with the debate modelling based on argumentation theory [4] to introduce the notion of attack between propositions or for the explanation [1] or more complex frameworks like contextual preference-based argumentation frameworks [2].

We have distinguished two configurations of agents in our work: collaborative agents and cooperative agents. In the case of cooperative agents, the problem of building an action plan satisfying the objectives of the agents is stated as a debate. The debate modelling is better suited to practical situations where each agent controls his/her own know-how, and only shares the part of his knowledge which is required for the common goal, defending his/her own interests and not necessarily revealing his/her weaknesses. The proposed model is based on fuzzy sets and MCDA techniques to represent the relationship between actions and objective satisfaction and to aggregate different points of view.

\section{References}

[1] L. Amgoud, H. Prade, Using arguments for making and explaining decisions, Artificial Intelligence 173 (3-4) (2009) 413-436.

[2] J.-R. Bourguet, L. Amgoud, R. Thomopoulos, Towards a unified model of preference-based argumentation, in: International Symposium on Foundations of Information and Knowledge Systems, Springer, 2010, pp. 326-344.

[3] G. B. Dagnino, Coopetition strategy: a new kind of interfirm dynamics for value creation, in: Coopetition Strategy, Routledge, 2009, pp. 45-63.

[4] P. M. Dung, On the acceptability of arguments and its fundamental role in nonmonotonic reasoning, logic programming and n-person games, Artificial Intelligence 77 (2) (1995) 321-357.
[5] R. Felix, Multicriteria decision making (mcdm): Management of aggregation complexity through fuzzy interactions between goals or criteria, in: Int. Conf. on Information Processing and Management of Uncertainty in Knowledge-Based Systems (IPMU), Malaga, Spain, 2008.

[6] M. Grabisch, S. Greco, M. Pirlot, Bipolar and bivariate models in multicriteria decision analysis: Descriptive and constructive approaches, International Journal of Intelligent Systems 23 (9) (2008) 930-969.

[7] A. Imoussaten, J. Montmain, F. Trousset, C. Labreuche, Multi-criteria improvement of options, in: Proceedings of the 7 th conference of the European Society for Fuzzy Logic and Technology, Atlantis Press, 2011, pp. 1030-1037.

[8] A. Imoussaten, F. Trousset, J. Montmain, Improving performances in a company when collective strategy comes up against individual interests, in: Proceedings of the 7th conference of the European Society for Fuzzy Logic and Technology, Atlantis Press, 2011, pp. 904-911.

[9] R. L. Keeney, H. Raiffa, Decisions with Multiple Objectives Preferences and Value Tradeoffs, Cambridge University Press, 1976.

[10] J. Montmain, C. Labreuche, A. Imoussaten, F. Trousset, Multi-criteria improvement of complex systems, Information Sciences 291 (2015) 6184 . 